\title{
Metamizol como inmunomodulador en el dolor neuropático
}

\author{
Metamizole as an immunomodulator in neuropathic pain
}

\section{Pérez Hernández}

Unidad del Dolor. Hospital Universitario de La Princesa, Madrid. Universidad Alfonso X el Sabio (UAX). Madrid, España

El dolor neuropático tiene una prevalencia entre el 4 y el $8 \%$ de la población, según diversos estudios. Clínicamente la intensidad del dolor es 2 puntos superior al dolor nociceptivo, con mayor repercusión sobre la calidad de vida, la ansiedad, el sueño y la depresión (1). Hasta un $37 \%$ de los procesos de lumbalgia crónica se asocian a radiculopatía, la causa más frecuente de dolor neuropático [2], siendo la causa más frecuente de dolor neuropático en las unidades de dolor en España (3).

La etiopatogenia varía según los diferentes procesos, pero existen fenómenos comunes muy específicos, como la neoexpresión de canales del Na+ Nav1.3, fenómenos de activación simpática, plasticidad neuronal medular, pérdida de mecanismos inhibitorios, y otros como la activación de la microglía, favoreciendo mecanismos inmunes de sensibilización central (4). Todo ello hace que los tratamientos hasta la actualidad tengan una eficacia limitada, a pesar de actuar sobre neurotransmisores o receptores de la trasmisión nociceptiva, siendo muy pocos los fármacos con indicación específica $[5,6]$, y esto contribuye a que los costes sociosanitarios sean elevados [7].

Los mecanismos inmunes cada vez están teniendo más protagonismo en la fisiopatología del dolor neuropático, y diversas citoquinas y quimioquinas parecen jugar un papel importante a nivel del sistema nervioso periférico y central por su efecto pronociceptivo [8]. Sabemos que en el ganglio de la raíz dorsal, donde se encuentra en núcleo celular, tiene lugar una gran actividad metabólica que rige la nocicepción. Se ha identificado en el GDR la producción múltiples citoquinas (TNF- $\alpha$, IL-1 $\beta$, IL3, IL5, IL6, II-8, IL17) y quimioquinas (CXCL1, 2, 3, 4, 5, 6) y sus respectivos receptores (9). También conocemos los mecanismos de transducción intracelular cuando estos medidores inmunológicos se unen a sus receptores, activando diversas vías de señalización como JAK/STAT, MAK quinasas o NF-K $\beta$, que activan en el núcleo la síntesis proteica. Esto se suele traducir en el aumento de la expresión de receptores para estas mismas citocinas y de noci- ceptores (como TRPV1,4] y canales iónicos del $\mathrm{Na}+$ $\mathrm{K}+$ y Ca++ (10). Los fenómenos de cronificación se asocian con la activación de la microglía en la médula espinal [11].

El cómo modular esta respuesta nociceptiva inmunomediada, tanto en el dolor neuropático como también en el inflamatorio, es una de las líneas de investigación actual.

Zajaczkowska y cols. acaban de publicar un trabajo con repercusión translacional, por su aplicabilidad clínica, donde estudian los efectos del metamizol en un modelo clásico de dolor neuropático por ligadura del ciático (12). Los resultados evidencian que la administración repetida intraperitoneal de metamizol disminuye el desarrollo de síntomas de dolor neuropático en ratas. Simultáneamente, el metamizol disminuye la expresión de interleucinas pronociceptivas (IL-1 beta, IL-6 e IL-18) y quimiocinas (CCL2, CCL4 y CCL7) en el GDR medido 7 días después de la lesión del nervio ciático. Estos datos indican que el metamizol ejerce efectos antinociceptivos sobre el dolor neuropático inducido por la lesión nerviosa a nivel de GRD. Este medicamento, comercializado desde 1922, se suele usar para aliviar el dolor somático y visceral pero no para tratar el dolor neuropático. A pesar de todo, sus mecanismos de acción analgésica no son bien conocidos, pero no parece tener un especial papel en la inhibición de las prostaglandinas, y en los últimos años algunos autores han dilucidado la importancia del bloqueo de los receptores TRPA1 en su mecanismo de acción (13). Hasta nuestro conocimiento, solo existe publicado un estudio clínico en lumbalgia/radiculopatía que comparaba la eficacia de metamizol versus diclofenaco y placebo, con superioridad para el metamizol en analgesia y funcionalidad; a pesar de ello es un ensayo clínico en el que las dosis son vía i.m., una sola vez al día y con una duración de 48 horas (14).

Otro fármaco como la dexmedetomidina también ha mostrado recientemente, en un modelo similar de constricción del ciático, la capacidad de bloquear el eje 
TLR4/NF-kB, y revertir la expresión de interleucinas IL-6, TNF- $\alpha$ e IL-8 (15).

Estos datos y otros adicionales están abriendo las posibilidades terapéuticas hacia mecanismos neuroinflamatorios, poniendo en evidencia el papel de los mismos en el dolor neuropático. Además, tenemos fármacos ya conocidos, como el metamizol o la desmetomidina, que podemos rentabilizar mejor.

\section{BIBLIOGRAFÍA}

1. Pérez C, Margarit C, Sánchez-Magro I, de Antonio A, Villoria J. Chronic Pain Features Relate to Quality of Life More than Physiopathology: A Cross-Sectional Evaluation in Pain Clinics. Pain Pract. 2017;17(7):866-78. DOI: 10.1111/papr.12533.

2. Baron R, Binder A, Wasner G. Neuropathic pain: diagnosis, pathophysiological mechanisms, and treatment. Lancet Neurol. 2010;9(8):807-19. DOI: 10.1016/S14744422(10)70143-5.

3. Pérez C, Ribera MV, Gálvez R, Micó JA, Barutell C, Failde I, et al. High prevalence of confirmed, but also of potential and believed, neuropathic pain in pain clinics. Eur J Pain. 2013;17(3):347-56. DOI: 10.1002/j.15322149.2012.00204.x

4. Colloca L, Ludman T, Bouhassira D, Baron R, Dickenson AH, Yarnitsky D, et al. Neuropathic pain. Nat Rev Dis Primers. 2017;3:17002. DOI: 10.1038/nrdp.2017.2.

5. Finnerup NB, Attal N, Haroutounian S, McNicol E, Baron $\mathrm{R}$, Dworkin RH, et al. Pharmacotherapy for neuropathic pain in adults: a systematic review and meta-analysis. Lancet Neurol. 2015;14(2):162-73. DOI: 10.1016/S14744422(14)70251-0.

6. Binder A, Baron R. The Pharmacological Therapy of Chronic Neuropathic Pain. Dtsch Arztebl Int. 2016;113(37):616-25. DOI: 10.3238/arztebl.2016.0616.

7. Pérez C, Navarro A, Saldaña MT, Wilson K, Rejas J. Modeling the predictive value of pain intensity on costs and resources utilization in patients with peripheral neuropathic pain. Clin J Pain. 2015;31(3):273-9. DOI: 10.1097/AJP. 0000000000000110.

8. Gonçalves Dos Santos G, Delay L, Yaksh TL, Corr M. Neuraxial Cytokines in Pain States. Front Immunol. 2020;10:3061. DOI: 10.3389/fimmu.2019.03061.

9. Cook AD, Christensen AD, Tewari D, McMahon SB, Hamilton JA. Immune Cytokines and Their Receptors in Inflammatory Pain. Trends Immunol. 2018;39(3):240-55. DOI: 10.1016/j.it.2017.12.003.

10. Skaper SD, Facci L, Zusso M, Giusti P. Neuroinflammation, Mast Cells, and Glia: Dangerous Liaisons. Neuroscientist. 2017;23(5):478-98. DOl: 10.1177/1073858416687249.

11. Cairns BE, Arendt-Nielsen L, Sacerdote P. Perspectives in Pain Research 2014: Neuroinflammation and glial cell activation: The cause of transition from acute to chronic pain? Scand J Pain. 2015;6(1):3-6. DOI: 10.1016/j.sjpain.2014. 10.002.

12. Zajaczkowska R, Kwiatkowski K, Pawlik K, Piotrowska A, Rojewska E, Makuch W, et al. Metamizole relieves pain by influencing cytokine levels in dorsal root ganglia in a rat model of neuropathic pain. Pharmacol Rep. 2020;10.1007/ s43440-020-00137-8. DOI: 10.1007/s43440-02000137-8.

13. Nassini R, Fusi C, Materazzi S, Coppi E, Tuccinardi T, Marone IM, et al. The TRPA1 channel mediates the analgesic action of dipyrone and pyrazolone derivatives. $\mathrm{Br} \mathrm{J}$ Pharmacol. 2015;172(13):3397-411. DOI: 10.1111/bph.13129.

14. Babej-Dölle R, Freytag S, Eckmeyer J, Zerle G, Schinzel S, Schmeider G, et al. Parenteral dipyrone versus diclofenac and placebo in patients with acute lumbago or sciatic pain: randomized observer-blind multicenter study. Int J Clin Pharmacol Ther. 1994;32(4):204-9.

15. Zhang W, Yu T, Cui X, Yu H, Li X. Analgesic effect of dexmedetomidine in rats after chronic constriction injury by mediating microRNA-101 expression and the E2F2/TLR4/NF-KB. Exp Physiol. 2020;10.1113/EP088596. DOI: 10.1113/ EP088596. 